\title{
A Case of Coil Embolization for a Ruptured Anterior Spinal Artery Aneurysm Associated with Bilateral Vertebral Artery Occlusion
}

\author{
Nobuhiko KawaI, ${ }^{1}$ Masaki TATANO, ${ }^{1}$ Ryoji ImOTo, ${ }^{1}$ Koji HiRAshita, ${ }^{1}$ \\ Masatoshi YUNOKI, ${ }^{1}$ and Kimihiro YOSHINO $^{1}$ \\ ${ }^{1}$ Department of Neurosurgery, Kagawa Rosai Hospital, Marugame, Kagawa, Japan
}

\begin{abstract}
Anterior spinal artery (ASA) aneurysms are rare, and the majority are associated with vascular lesions such as arteriovenous malformations, moyamoya disease, and aortic stenosis. Herein, we report a case of a ruptured anterior spinal artery aneurysm caused by bilateral vertebral artery (VA) occlusion, which was treated by coil embolization. An 83-year-old man was found collapsed at home, and was brought in by emergency. His consciousness level was I-1 on the Japan Coma Scale, and there were no symptoms such as paralysis in the extremities. Computed tomography showed Fisher 3 subarachnoid hemorrhage, while magnetic resonance angiography showed an aneurysm in the right VA. Digital subtraction angiography showed bilateral VA occlusion, and an aneurysm was found on the dilated ASA as a collateral circulation. Coil embolization was performed after confirmation of no hemodynamic problems. No postoperative adverse events were observed. Coil embolization may be an effective treatment for ruptured aneurysms of the ASA.
\end{abstract}

Keywords: anterior spinal artery, aneurysm, coil embolization, ruptured aneurysm, vertebral artery occlusion

\section{Introduction}

Anterior spinal artery (ASA) aneurysms are rare, and most of them are associated with vascular lesions such as arteriovenous malformations, Moyamoya disease, aortic coarctation, etc. The treatment strategy for ASA aneurysms has not reached consensus, which is currently determined on a case-by-case basis. In this manuscript, we experienced a case of a ruptured aneurysm in the ASA that dilated as a collateral flow, which was caused by bilateral vertebral artery (VA) occlusion. In this case, a microcatheter was guided through the dilated ASA into the aneurysm, and coil embolization was performed.

\section{Case Report}

The case was an 83-year-old man scheduled for surgery and radiation therapy for laryngeal cancer.

Received June 3, 2020; Accepted September 28, 2020

Copyright $\subseteq 2021$ by The Japan Neurosurgical Society This work is licensed under a Creative Commons AttributionNonCommercial-NoDerivatives International License.
He was found unconscious and transported to our hospital by ambulance. His consciousness level at consultation was I-1 on the Japan Coma Scale. No other neurological symptoms were present. Computed tomography (CT) showed a Fisher 3 subarachnoid hemorrhage (Fig. 1A). Magnetic resonance angiography (MRA) revealed abnormal vascular lesion (Fig. 1B), which was suspected to be a bleeding point. The MRA at 8 years prior to this case showed the right VA was stenotic and left VA was patent (Fig. 1C). Digital subtraction angiography (DSA) showed an aneurysm at the intracranial junction of the dilated ASA (Figs. 2A and 2B), which was irregularly shaped, and a broad neck aneurysm $(6.0 \mathrm{~mm} \times 5.0 \mathrm{~mm})$. The bilateral VAs were occluded, and the basilar artery communicated with the right VA via the anterior radicular artery and ASA.

A 5Fr ENVOY guiding catheter (Johnson \& Johnson, New Brunswick, NJ, USA) was placed in the right subclavian artery. Because the anterior radicular artery was too thin to place a distal access catheter, an Excelsior SL-10 microcatheter (Stryker, Kalamazoo, MI, USA) was inserted into the right VA and navigated into the ASA. It was difficult to insert the microcatheter because there was a large curve in 

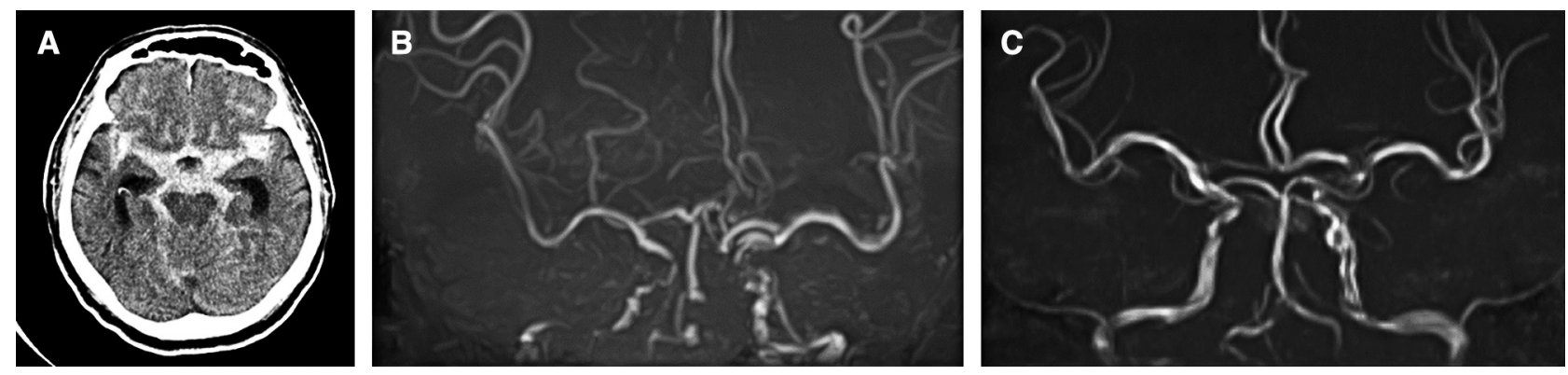

Fig. 1 (A) Axial head CT scan revealed a subarachnoid hemorrhage (Fisher grade 3). (B) MRA of the onset. The image shows an aneurysm and occluded bilateral VA. (C) MRA taken 8 years prior at our hospital showed bilateral VA, but with severe stenosis in the proximal VA. CT: computed tomography, MRA: magnetic resonance angiography, VA: vertebral artery.

the anterior radicular artery where it entered the spinal canal. When we placed Excelsior SL-10 microcatheter in the ASA and imaged the aneurysm, it was considered that antegrade blood flow was maintained because the contrast agent did not accumulate on the distal side of the aneurysm. However, the contrast effect was so thin that it was not possible to create a roadmap from the ASA to the aneurysm. For this reason, we used normal images while approaching the aneurysm. When the tip of the Excelsior SL-10 microcatheter was placed into position and the aneurysm was imaged again, the basilar artery was visualized with the aneurysm, and it was confirmed that no contrast agent remained (Fig. 3A).
Therefore, we thought that blood flow was secured within the ASA. The aneurysm was occluded with coils (ED coil, $3 \mathrm{~mm} \times 6 \mathrm{~cm}$; ED coil, $2 \mathrm{~mm} \times 4 \mathrm{~cm}$; Ultrasoft, $1.5 \mathrm{~mm} \times 2 \mathrm{~cm}$; Kaneka Medix, Osaka, Japan) with preservation of ASA patency. The coil part of the aneurysm was not imaged, whereas the bleb was imaged with a slight delay (Fig. 3B).

Following the procedures described above, dual antiplatelet therapy $(100 \mathrm{mg} /$ day of aspirin and $75 \mathrm{mg} /$ day of clopidogrel) was started. No postoperative consciousness deterioration or paralysis was observed. DSA performed 3 months later confirmed the patency of the ASA and disappearance of the aneurysm (Fig. 3C). Magnetic resonance imaging at
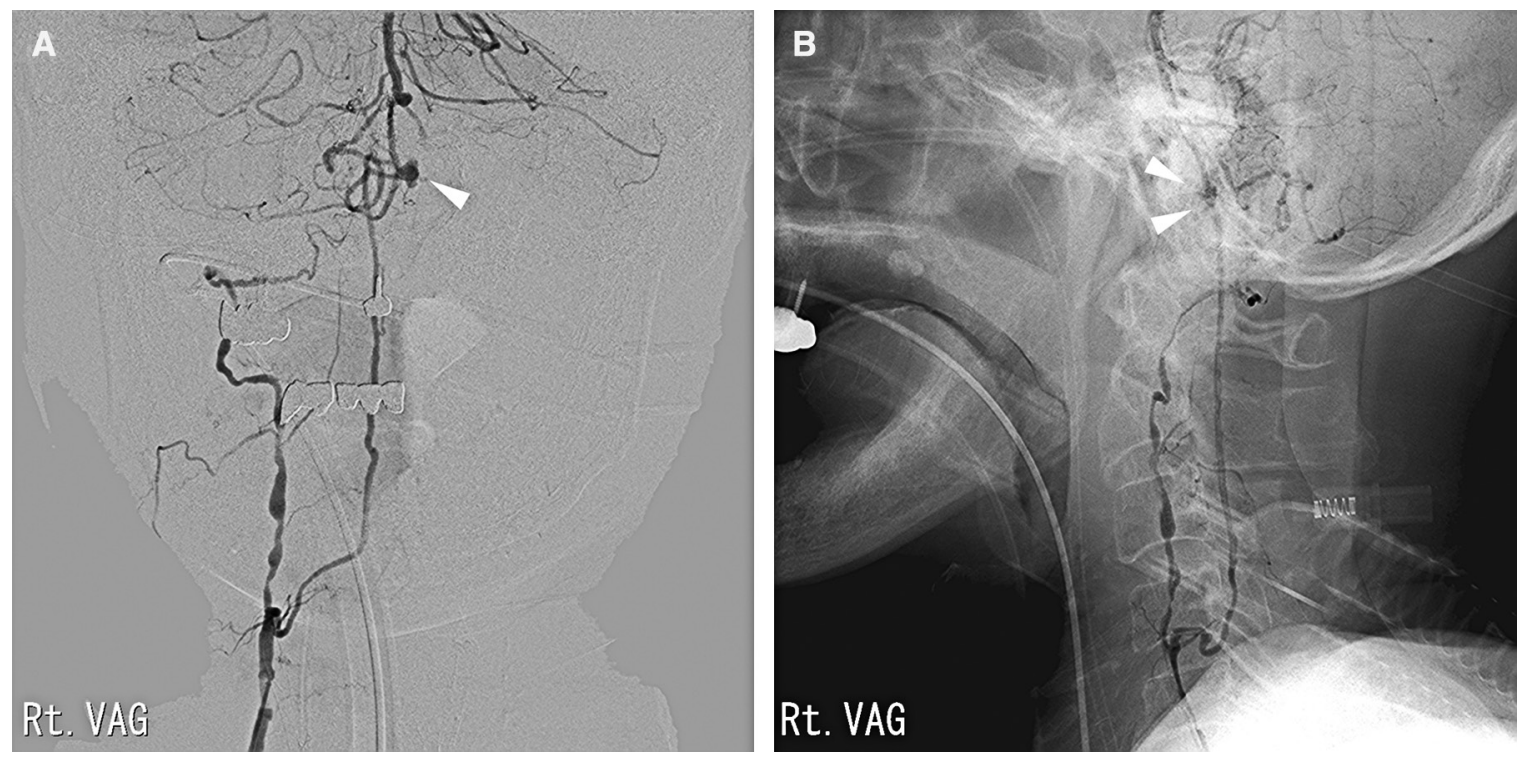

Fig. 2 (A) A-P and (B) lateral views of the right vertebral angiography on admission. High-grade atherosclerotic wall irregularities were found. The right vertebral artery was occluded at the extracranial segment while the ASA bifurcated at the level of the fourth vertebral body. The ASA was dilated and perfused the circulation area of the vertebral basilar artery. An aneurysm was noted at the intracranial portion of the dilated ASA (arrow head). ASA: anterior spinal artery. 

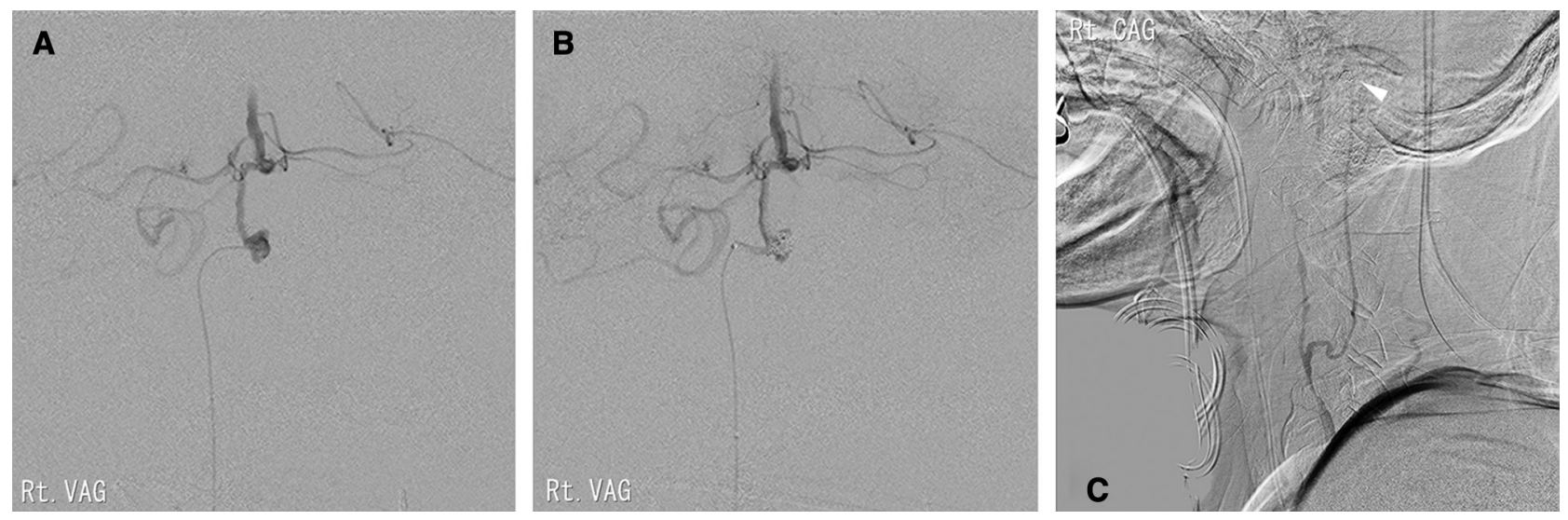

Fig. 3 (A) A-P view. The microcatheter tip was introduced into the aneurysm, and angiography was performed. Right vertebral angiography was performed after coil embolization. (B) After embolization of the aneurysm, adequate blood flow of the vertebrobasilar artery was confirmed. (C) Lateral views of the right vertebral angiography after 3 months. The arrowhead is the part where the aneurysm was not visualized.

Table 1 Reported cases of ruptured ASA aneurysms associated with VA occlusion

\begin{tabular}{|c|c|c|c|c|c|}
\hline Author (year) & Age/sex & Occluded VA & $\begin{array}{c}\text { Location of } \\
\text { aneurysm }\end{array}$ & Surgery & Prognosis \\
\hline Kawamura S (1999) & $42 / \mathrm{M}$ & bil VA & Posterior fossa & clipping & No deficit \\
\hline Kitayama M (2008) ${ }^{3)}$ & $71 / \mathrm{M}$ & bil VA & $\mathrm{C} 2-3$ & clipping & Bed ridden \\
\hline Karakama J (2010) ${ }^{8)}$ & $51 / \mathrm{M}$ & rt VA & C1 & not performed & No deficit \\
\hline Yoshida M (2012) & 66/M & rt VA & Posterior fossa & clipping & No deficit \\
\hline Ashour R (2015) ${ }^{1)}$ & No data & bil VA & $\mathrm{C} 2$ & Clipping & No deficit \\
\hline Present case & $83 / \mathrm{M}$ & bil VA & Posterior fossa & Coil embolization & Bed ridden \\
\hline
\end{tabular}

ASA: anterior spinal artery; VA: vertebral artery

1 and 3 months of follow-up confirmed no new cerebral infarctions.

\section{Discussion}

Ruptured ASA aneurysms are rare. Aneurysms may occur and rupture because of hemodynamic stress, such as arteriovenous malformation and aortic stenosis, ${ }^{1,2)}$ following autoimmune diseases, connective tissue diseases, and mycosis, as well as because of fragility of the vessel wall. ${ }^{3-6)}$

To our knowledge, five authors reported ruptured ASA aneurysms associated with VA occlusion (Table 1). ${ }^{1,3,5,7,8)}$ The age range of these cases was $42-83$ years; four were bilateral, and two had unilateral VA occlusion. These findings suggest that VA occlusion may be involved in the formation of ASA aneurysms.

We presumed that the aneurysm was occurred by a hemodynamic mechanism associated with occlusion of bilateral VAs. Because the aneurysm had bleb and were irregular shaped, which was likely to re-rupture, we planned treatment for that patient.
There is no consensus about the treatment of ASA aneurysms. ${ }^{5}$ Some previous reports showed that craniotomy has good results for ruptured ASA caused by hemodynamic pathology., ${ }^{5,9,10)}$ However, Kawamura et al. ${ }^{7)}$ reported a case, where pre-rupture spinal artery aneurysm caused by unilateral VA occlusion was treated conservatively and eventually disappeared.

It was considered that direct surgery was not suitable for him because the patient was elderly. There are no other reports of endovascular treatment for rupture intracranial ASA aneurysm. However, in this case, endovascular treatment was considered less invasive than craniotomy because the device approach to the aneurysm was seemed to be relatively easy. ${ }^{11)}$

When we performed endovascular treatment, there were some points that made us difficult to judge. The first point was how to maintain blood flow of the ASA. In our case, the artery in which the aneurysm was occurring was thin, and there was not much blood flow. Guiding catheters and 
intermediate catheters should be placed more distally to improve the operability of microcatheter. However, in this case, we judged that inserting a device thicker than the microcatheter into the ASA could occlude the blood vessels; therefore, we moved only the microcatheter upward from the origin of the ASA.

The second point was that it might be difficult for us to take a detailed roadmap when the ASA were thin and there was not much blood flow. In most cases, ASA is thin and has little blood flow; thus, it is difficult to visualize it by angiography. In this case, the ASA was dilated as a collateral circulation; therefore, it was possible to visualize the artery by angiography. However, the contrast effect was insufficient due to the strong stenosis of the right VA, and thus blood flow to the ASA was poor. For this reason, we treated the aneurysm without using a roadmap. Fortunately, from the right VA to the aneurysm, there was no branching artery which caused trouble when judging the progress of the microcatheter, and it was a straight road. Therefore, it was possible to operate the microcatheter inside the ASA.

The final point was the embolization of the aneurysm was terminated intentionally incomplete. Because this aneurysm had an irregular shape and its size was not so large, there was a risk of rupture if the coil was used more than necessary. Moreover, thrombosis and thrombotic occlusion due to the coil being ejected into the ASA due to excessive placement of the coil have been feared. It was judged that ASA had not have much blood flow, and thrombosis in the aneurysm might be promoted even with incomplete occlusion on imaging. As a result, post-treatment angiography showed disappearance of the aneurysm without neck remnant.

When embolizing an aneurysm, we need to accurately evaluate its shape and hemodynamics.

In conclusion, ASA aneurysm can be safely treated with endovascular treatment if the blood flow of ASA is maintained sufficiently during the device manipulate in the ASA.

\section{Conflicts of Interest Disclosure}

All authors confirm that they have no conflicts of interest.

\section{References}

1) Ashour R, Filippidis A, Patel N: Ruptured anterior spinal artery aneurysm associated with bilateral vertebral artery occlusion treated by surgical clipping. World Neurosurg 84: 1178.e11-3, 2015

2) Walz DM, Woldenberg RF, Setton A: Pseudoaneurysm of the anterior spinal artery in a patient with Moyamoya: an unusual cause of subarachnoid hemorrhage. AJNR Am J Neuroradiol 27: 1576-1578, 2006

3) Kitayama M, Nishioka K, Okawa T, et al.: Subarachnoid hemorrhage due to rupture of an anterior spinal artery aneurysm: a case report. Spinal Surgery 23: 91-94, 2008

4) Klingler JH, Gläsker S, Shah MJ, Van Velthoven V: Rupture of a spinal artery aneurysm attributable to exacerbated Sjögren syndrome: case report. Neurosurgery 64: E1010-1011; discussion E1011, 2009

5) Yoshida $M$, Ichihara $K$, Nakabayashi $K$, et al.: A case of subarachnoid hemorrhage due to intracranial anterior spinal artery aneurysm. Surg Cereb Stroke 40: 135-139, 2012 (Japanese)

6) Matsui T, Taniguchi T, Saitoh T, et al.: Hematomyelia caused by ruptured intramedullary spinal artery aneurysm associated with extramedullary spinal arteriovenous fistula-case report. Neurol Med Chir (Tokyo) 47: 233-236, 2007

7) Kawamura S, Yoshida T, Nonoyama Y, Yamada M, Suzuki A, Yasui N: Ruptured anterior spinal artery aneurysm: a case report. Surg Neurol 51: 608-612, 1999

8) Karakama J, Nakagawa K, Maehara T, Ohno K: Subarachnoid hemorrhage caused by a ruptured anterior spinal artery aneurysm. Neurol Med Chir (Tokyo) 50: 1015-1019, 2010

9) Berlis A, Scheufler KM, Schmahl C, Rauer S, Götz F, Schumacher M: Solitary spinal artery aneurysms as a rare source of spinal subarachnoid hemorrhage: potential etiology and treatment strategy. AJNR Am J Neuroradiol 26: 405-410, 2005

10) Gonzalez LF, Zabramski JM, Tabrizi P, Wallace RC, Massand MG, Spetzler RF: Spontaneous spinal subarachnoid hemorrhage secondary to spinal aneurysms: diagnosis and treatment paradigm. Neurosurgery 57: 1127-1131; discussion 1127-1131, 2005

11) Lavoie P, Raymond J, Roy D, Guilbert F, Weill A: Selective treatment of an anterior spinal artery aneurysm with endosaccular coil therapy. Case report. J Neurosurg Spine 6: 460-464, 2007

Corresponding author: Kimihiro Yoshino, MD, PhD Department of Neurosurgery, Kagawa Rosai Hospital, 3-3-1 Jotocho, Marugame, Kagawa 763-8502, Japan. e-mail: yoshino@kagawaH.johas.go.jp 